\title{
Determinación de restricciones de capacidad de producción en proceso de obtención de cobre
}

\author{
Determination of production capacity constraints \\ in the process of obtaining copper \\ Jaime Núñez Segovia $^{1 *} \quad$ Johann Godoy Garvs $^{1} \quad$ Luis Pérez Pozo $^{1}$ \\ Recibido 11 de enero de 2016, aceptado 18 de abril de 2016 \\ Received: January 11, 2016 Accepted: April 18, 2016
}

\begin{abstract}
RESUMEN
El trabajo consiste en un estudio de Simulación de Eventos Discretos (DES) para determinar las restricciones de capacidad de producción ("cuello de botella") en el proceso de conminución de mineral de cobre sulfurado en una planta minera en Chile y cómo estas restricciones cambian bajo distintos escenarios de operación. El modelo de simulación del proceso de conminución comprende las etapas de chancado, molienda SAG, primaria, secundaria, pebbles y de espesamiento.

El objetivo principal de la investigación es determinar los equipos "cuellos de botella" en el proceso mediante un estudio DES que comprende la modelación de la planta y la experimentación de distintos escenarios de operación utilizando el Sw SIMIOß. La metodología del estudio de simulación sigue las etapas propuestas por Kelton y las recomendaciones presentes en la literatura. Para determinar las restricciones de capacidad ("cuellos de botella" principal, secundarios y cambiantes) se emplea el método de Utilización. Los resultados incluyen el modelo DES validado, alcanzando en la simulación de la variable principal de resultado, un error relativo de $0,66 \%$, un error porcentual absoluto medio de $2,67 \%$ y una señal de seguimiento del error dentro de \pm 3 DAM. Se determinaron los equipos "cuellos de botella" para la condición histórica de operación de la planta en 2014 y su comportamiento en los distintos escenarios de operación ensayados.
\end{abstract}

Palabras clave: DES, conminución, “cuellos de botella”, modelamiento, SIMIO®.

\begin{abstract}
This work is a study of Discrete Event Simulation (DES) to determine the constraints of production capacity "bottleneck" in the process of comminution at a copper sulphide ore processing plant in Chile and how these restrictions change under different operating scenarios. Steps of crushing, grinding (primary, secondary and pebbles) and thickening tank are comprised in the simulation model of the process.

The main objective of this research is to determine "bottlenecks" in the ore comminution process through a DES study that includes plant modeling and testing of different operating scenarios using the SIMIO® Sw. The phases proposed by Kelton and literature recommendations are followed by simulation study methodology. Utilization method is used to determine capacity constraints and "bottlenecks" (main, secondary, and changing). A validated model is achieved. The main outcome shows a relative error of $0.66 \%$, a mean absolute percentage error of $2.67 \%$ and a tracking error signal within \pm 3 DAM. Bottlenecks are identified for the historical performance of operation in the plant in 2014. The performance of the identified "bottlenecks" is tested under different operation scenarios.
\end{abstract}

Keywords: DES, comminution, "bottleneck", modelling, SIMIO®.

1 Departamento de Ingeniería Mecánica. Universidad Técnica Federico Santa María (UTFSM). Casilla 110-V. Valparaíso, Chile. E-mail: jaime.nunez@usm.cl

* Autor de correspondencia 


\section{INTRODUCCIÓN}

La minería del cobre está sometida a crecientes presiones para elevar sus niveles de eficiencia y productividad de sus procesos y lograr así estructuras de costos que le permitan operar con márgenes de utilidad adecuados y sostenibles en el tiempo. Detectar las restricciones de capacidad de sus procesos es una ventaja que permite a las empresas verificar el plan de producción anual, bajo las condiciones presentes en su sistema productivo y tomar mejores decisiones en su operación, mantenimiento e inversiones.

El uso de modelos de simulación computacional para entender el funcionamiento de un sistema, realizar experimentos para evaluar diferentes estrategias y mejorar la eficiencia de estos, es una opción de probada efectividad en gran variedad de entornos de producción [1-2]; en particular, DES tiene la capacidad de imitar la dinámica y complejidad de un sistema real [3].

El modelado de sistemas industriales reales, como es el caso de una planta de conminución de cobre, involucra procesos discretos y continuos, que requiere de enfoques adecuados para manejar esta complejidad. Estos enfoques de simulación están extensamente discutidos en la literatura especializada [1, 3-7]. Los sistemas mixtos, discreto-continuo, característicos de procesos de manipulación de materiales a granel, de líquidos, y de pulpa de mineral, entre otros, son difíciles de modelar debido a la presencia de aleatoriedad y eventos discretos, que no interaccionan fácilmente con ecuaciones algebraicas y diferenciales y, por otro lado, debido a la presencia de tasas de flujo que son variables continuas [2, 5, 8-9].

Para efectuar estudios de simulación en sistemas reales, en la literatura especializada [2, 4, 7, 10- 11] se reconocen las siete etapas siguientes: diseño conceptual; análisis de las entradas del sistema; desarrollo del modelo; verificación; validación; análisis de las salidas; y experimentación con el modelo de simulación.

La experimentación con modelos DES, permite caracterizar el comportamiento y determinar limitaciones de capacidad en plantas y procesos, entre otros usos.
Dentro de las técnicas para determinar, analizar y gestionar los recursos que limitan la capacidad de sistemas discretos y discretos-continuos, Roser [12] resume las principales características de cinco métodos de detección de "cuellos de botella". Ejemplos de técnicas para análisis de "cuellos de botella" y sus resultados, se documentan en: Sengupta [13] identifica y clasifica limitantes de capacidad en un sistema de fabricación; Dewa [14] demuestra que un sistema manual de montaje de automóviles puede ser optimizado por medio de la gestión de los "cuellos de botella"; Williams [15] ofrece un enfoque para entender la relación entre los "cuellos de botella", el retraso acumulado y el flujo de una red ferroviaria, con el fin de apoyar las decisiones de operación e inversión en capacidad; Sharda [16] realiza reducción de "cuellos de botella" en diferentes productos en una planta química, utilizando un enfoque general de análisis en sistemas discreto-continuos.

Por otra parte, Roser [17] describe un método para detectar y vigilar los "cuellos de botella" y el desplazamiento de estos "cuellos de botella" en sistemas de producción, en estado estacionario y no estacionario sujeto a variación aleatoria; asumiendo que todos los sistemas de fabricación se ven limitados por uno o más "cuellos de botella", y que estos se pueden "desplazar" de una máquina a otra, debido al cambio del sistema con el tiempo, o debido a eventos aleatorios.

Lima [18] presenta una metodología de selección del método más apropiado de detección de "cuellos de botella" en un sistema real, aplicada a un proceso real de empresa química, que es adecuada incluso para sistemas con los "cuellos de botella" cambiantes.

\section{ANTECEDENTES DE LA INVESTIGACIÓN}

A continuación se presenta la descripción de la Planta de Conminución donde se realiza la investigación, los objetivos, las preguntas de investigación, la metodología del trabajo y el modelo de simulación desarrollado.

\section{Descripción de la Planta}

La Planta de Conminución de Cobre sulfurado en estudio fue diseñada para una capacidad de procesamiento de 87.000 toneladas de mineral por día y comprende etapas de chancado y molienda. 
Como se observa en la Figura 1, la etapa de Chancado está compuesta por dos chancadores primarios giratorios de $60 \times 89$ [in], capaces de procesar $6.000[\mathrm{t} / \mathrm{h}]$ de mineral (ROM) proveniente de la mina. El mineral proveniente del Chancador 2 se conduce mediante el alimentador CM1 A a la correa transportadora CM2A y el mineral proveniente del Chancador 1 se traspasa mediante el alimentador CM1 a la correa transportadora CM2. Por medio de una Torre de Trasferencia se distribuye el mineral a la correa CM3, para alimentar al Acopio B y a través de la correa CVB003, se conduce el mineral a la Torre de Traspaso y posteriormente a la correa overland CVB004 que lo transporta al Acopio C para alimentar la etapa de Molienda de esta Planta.

En la etapa de Molienda, el mineral grueso se recupera de la reserva mediante alimentadores de cinta, y por medio de la correa CVB007 ingresa al circuito de molienda, donde la Molienda Primaria se realiza en un molino SAG de 22.000 [kW]. La Molienda Secundaria se realiza en dos molinos de bolas de $16.390[\mathrm{~kW}]$ cada uno, que funcionan en paralelo.

La descarga del Molino SAG "trommel undersize", y de los molinos de bolas se introduce en el Cajón de Descarga de molienda "Cuba". Cuatro Bombas centrifugas de $9.500\left[\mathrm{~m}^{3} / \mathrm{h}\right]$ de capacidad (dos operando y dos de reserva), suministran la pulpa desde la Cuba al circuito de Molienda Secundaria, aquí cada Molino de bolas opera en un recorrido cerrado con un grupo de dieciséis Ciclones de 838 [mm] de diámetro.

La descarga del Molino SAG "trommel oversize" se transfiere a dos Harneros vibrantes (uno operando y uno en espera). El tamaño de mineral inferior, se deriva a la Cuba y el sobre tamaño de mineral o Pebbles, es enviado al circuito de molienda de Pebbles por medio de las correas CVB100, CVH101, CVH102 y CVB103. La molienda de Pebbles se compone de la Tolva de Pebbles de 300 [t] que alimenta a dos Chancadores de Cono, cada uno con $750[\mathrm{~kW}]$ de potencia. El mineral triturado es enviado nuevamente a los molinos de bolas de Molienda Secundaria por medio de las correas transportadoras CVB104, CVB105, CVB106, CVB107 y CVB108.

El desbordamiento "overflow" del conjunto Ciclón, se deriva a través de los Harneros "desripeadores" al
Cajón Alimentación Espesadores que tiene la función de absorber las variaciones producción de la planta y alimentar a dos Espesadores, de 90 [m] de diámetro, cada Espesador posee un circuito de recirculación compuesto de una Bomba de recirculación de 725 $\left[\mathrm{m}^{3} / \mathrm{h}\right]$ de capacidad. La descarga "underflow" de los espesadores se conduce al Cajón Descarga Espesadores, que proporciona una pulpa con $60 \%$ de sólidos que es transportada por una tubería a la Flotación.

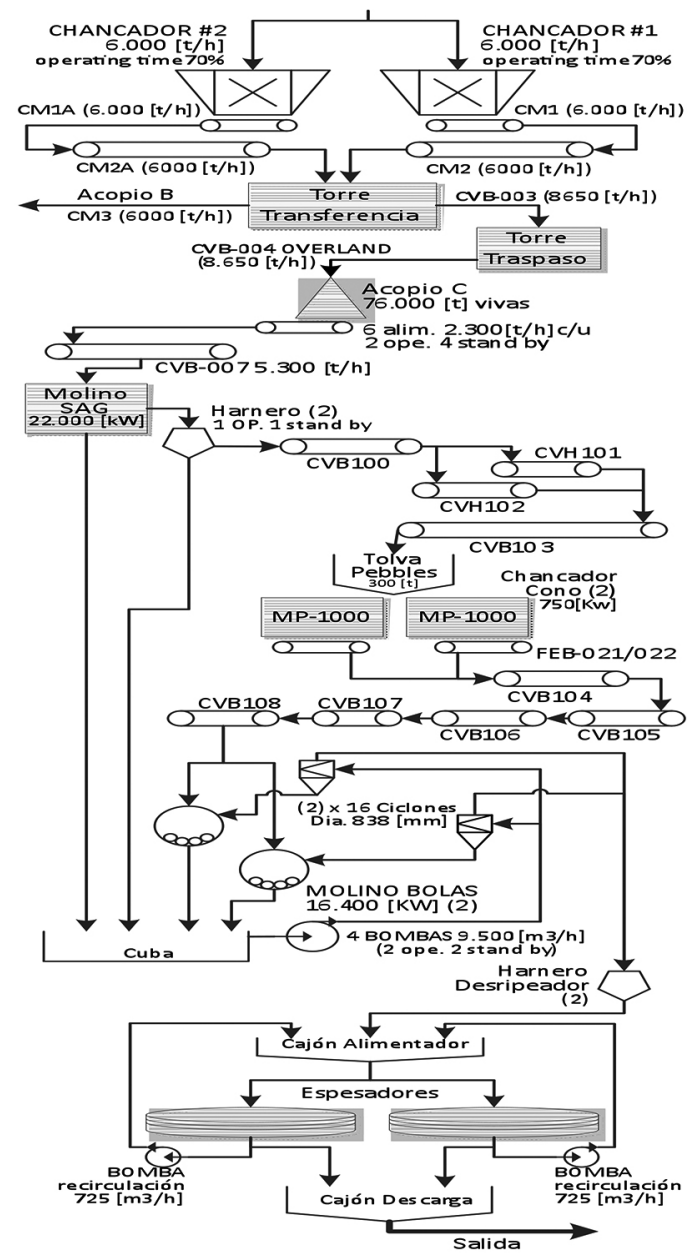

Figura 1. Diagrama de la Planta Conminución de Mineral.

\section{Objetivos}

El objetivo principal de la investigación es determinar los equipos "cuellos de botella" en el proceso de conminución de mineral de cobre, mediante un estudio DES que comprende la modelación de la 
planta y la experimentación de distintos escenarios de operación, utilizando el Sw SIMIO®.

Como objetivos secundarios se consideran: a) validar el modelo computacional de simulación del proceso utilizando la data de operación real de la empresa del 2014 con el fin de tener un modelo de experimentación creíble, que ayude a la toma de decisiones operacionales; b) determinar el comportamiento de los equipos "cuello de botella" por medio de la experimentación de distintas condiciones o escenarios de operación.

\section{Preguntas de investigación}

La experimentación es guiada por las necesidades de información de la unidad de planificación de la empresa y se traducen en las siguientes preguntas de investigación: 1) ¿cuáles son los equipos "cuellos de botella" de la Planta en la situación actual de operación?; 2) ¿cuáles son los equipos "cuellos de botella" de la Planta para distintos niveles de alimentación de mineral a la molienda SAG?; 3) ¿cómo afecta la proporción de Pebbles circulantes en la aparición de "cuellos de botellas"?; y 4) ¿cómo cambia la situación de los equipos "cuellos de botella" producto de variaciones en la capacidad de los chancadores primarios?

\section{METODOLOGÍA}

El estudio de simulación sigue la estructura y etapas de un proyecto de simulación formal [2].

El levantamiento del proceso incluye: descripción de procesos y equipos; recolección de la data de operaciones; lógicas de distribución de mineral, detención y reanudación de los equipos de la plantas.

El modelado conceptual considera la definición de los elementos del modelo y sus interrelaciones; los supuestos y simplificaciones realizados; variables de entrada y salida del modelo; el objetivo, límites y alcances del modelo; nivel de detalle; horizonte de simulación y escenarios de experimentación [7].

Se contempla la codificación en el Sw SIMIO® (Simio Enterprise Edition, propiedad de la empresa) [19-20]. El tratamiento del transporte continuo de material mediante cintas transportadoras, utiliza el enfoque propuesto en Fioroni [9] y Coelho [21], tratando el flujo como una serie de "porciones", lo que permite resultados estadísticamente similares al comportamiento real. La "discretización" de los flujos considera la relación directa entre el volumen transportado y el tiempo dedicado.

La verificación y validación del modelo busca comprobar que la simulación represente fielmente la realidad de operación de la Planta, usando la experiencia del personal de la empresa y datos de operación de 2014. Asimismo, se analizan las variables de resultado relevantes, usando estadísticos de media e intervalos de confianza para la estimación [22-23] y métricas para evaluar el error de pronóstico (relativo, absoluto medio y señal de seguimiento) de las variables de resultado con respecto a los datos reales del período 2014, usando un enfoque de pronóstico de series de tiempo [11,24].

Se considera la realización de experimentos para responder las preguntas de investigación y determinar las restricciones de capacidad mediante el método de Utilización [12, 17-18].

Se determina la línea base de existencia de "cuellos de botella" según las condiciones de operación actual de la Planta, y se diseñan tres tipos de experimentos monofactoriales, con distintos niveles cada factor, señalados en la Tabla 1, que permiten concluir a cerca de la existencia de "cuellos de botella" bajo distintos escenarios de operación.

\section{Modelo de simulación}

Los aspectos relevantes del modelo ChancadoMolienda logrado mediante el software de simulación SIMIO $®$, tiene las siguientes características: el modelo de simulación comienza con la creación de entidades denominadas "Mineral", generadas por una "Fuente" y luego transportadas mediante camiones hasta la Tolva de Alimentación de los Chancadores.

\section{Etapa de Chancado en el modelo de simulación}

La etapa de Chancado se representa mediante un modelo dinámico de cambio discreto compuesto por los subsistemas: Chancado que incluye la Tolva de Alimentación, Chancador primario y Tolva de Compensación; el subsistema Traspaso incluye la Torre de Transferencia y correas CM1, CM2, CM3, CM1A, CM2A, CVB003 y CVB004; y el subsistema Acopio incluye el Acopio B y el Acopio $\mathrm{C}$ de mineral y sus alimentadores. 
Tabla 1. Experimentos, factores y niveles.

\begin{tabular}{|c|c|c|}
\hline Experimento & Factor & Nivel \\
\hline \multirow{6}{*}{$\begin{array}{l}\text { 1. \% Utilización } \\
N^{\circ} \text { Réplicas: } 10 \\
\text { Período: } 365 \text { días } \\
\text { Discretización: } 20 \text { [t/u] }\end{array}$} & \multirow{6}{*}{$\begin{array}{c}\text { Alimentación } \\
\text { Molino SAG } \\
{[\mathrm{t} / \mathrm{h}]}\end{array}$} & $3.790+840 * \operatorname{Beta}(1,4 ; 1,13) ;$ Media: $4.270[\mathrm{t} / \mathrm{u}]$ \\
\hline & & $3.816[\mathrm{t} / \mathrm{h}]$ \\
\hline & & $3.900[\mathrm{t} / \mathrm{h}]$ \\
\hline & & $4.100[\mathrm{t} / \mathrm{h}]$ \\
\hline & & $4.390[\mathrm{t} / \mathrm{h}]$ \\
\hline & & $4.500[\mathrm{t} / \mathrm{h}]$ \\
\hline \multirow{6}{*}{$\begin{array}{l}\text { 2. \% Utilización } \\
\mathrm{N}^{\circ} \text { Réplicas: } 10 \\
\text { Período: } 365 \text { días } \\
\text { Discretización: } 20 \text { [t/u] }\end{array}$} & \multirow{6}{*}{$\begin{array}{l}\text { Porcentaje } \\
\text { Pebbles } \\
\text { circulante }\end{array}$} & $\operatorname{Normal}(9,11 ; 2,66) ;$ Media: $9,11 \%$ \\
\hline & & Normal $(15 ; 2,66) ;$ Media: $15 \%$ \\
\hline & & $\operatorname{Normal}(20 ; 2,66) ;$ Media: $20 \%$ \\
\hline & & $\operatorname{Normal}(25 ; 2,66) ;$ Media: $25 \%$ \\
\hline & & Normal $(30 ; 2,66) ;$ Media: $30 \%$ \\
\hline & & Normal $(9,11 ; 2,66) ;$ Media: $9,11 \%$ \\
\hline \multirow{7}{*}{$\begin{array}{l}\text { 3. \% Utilización } \\
\mathrm{N}^{\circ} \text { Réplicas: } 10 \\
\text { Período: } 365 \text { días } \\
\text { Discretización: } 20 \text { [t/u] }\end{array}$} & \multirow{7}{*}{$\begin{array}{l}\text { Capacidad } \\
\text { Chancador } \\
\text { Primario }\end{array}$} & $6.000[\mathrm{t} / \mathrm{h}]$ \\
\hline & & $5.500[\mathrm{t} / \mathrm{h}]$ \\
\hline & & $5.000[\mathrm{t} / \mathrm{h}]$ \\
\hline & & $4.600[\mathrm{t} / \mathrm{h}]$ \\
\hline & & $4.300[\mathrm{t} / \mathrm{h}]$ \\
\hline & & $4.000[\mathrm{t} / \mathrm{h}]$ \\
\hline & & $3.700[\mathrm{t} / \mathrm{h}]$ \\
\hline
\end{tabular}

Los Chancadores procesan el mineral y luego, mediante correas transportadoras y una lógica de operación definida en la Torre de Transferencia, se distribuye en los distintos acopios.

\section{Etapa de Molienda en el modelo de simulación} La etapa de Molienda se representa mediante un modelo dinámico de cambio discreto-continuo, compuesto por los subsistemas: Molienda Primaria que incluye el Molino SAG, Harnero y Correa CVB007; Molienda Secundaria que incluye el Cajón Descarga SAG, bombas de ciclones, ciclones, molinos de bolas y harneros desripeadores; Circuito de Pebbles que incluye la Tolva de Pebbles, chancadores de Pebbles y correas trasportadoras; y Espesamiento que incluye el Cajón de Descarga Espesadores y Espesador 1 y 2.

Los subsistemas Molienda Primaria y Circuito de Pebbles, son modelado mediante un modelo discreto. La entidad es generada en el acopio y por medio de alimentadores y la correa CVB007, transportada hasta el Molino SAG. Mediante una lógica operacional definida en el modelo, la entidad es descargada del Harnero y enviada al circuito de Pebbles (mineral sobre-tamaño) o a la Molienda Secundaria (mineral bajo-tamaño).
La Molienda Secundaria utiliza un modelo continuodiscreto. La entidad mineral proveniente del bajo tamaño del Harnero es transformado a un flujo continuo y acumulado en un estanque (Cajón Descarga $\mathrm{SAG}$ ). Dos bombas proporcionan un flujo continuo que es enviado a los ciclones. Mediante una lógica de operación definida en el modelo, el sobretamaño del ciclón es enviado a los molinos de bolas en conjunto con el mineral proveniente del Circuito de Pebbles, y el bajo tamaño del ciclón, es enviado a los harneros desripeadores y luego a los espesadores de mineral.

El Espesamiento y la tubería hacia la planta de concentrado, también es modelada mediante un modelo continuo-discreto.

\section{Procedimiento ejecución modelo}

Suponiendo que el usuario final de este prototipo es el trabajador operativo, la animación del proceso asume importancia, ya que permite ver lo que está sucediendo en el sistema, así como todas las decisiones y tráfico del mineral. Esto permite identificar qué sistema se está convirtiendo en "cuellos de botella". La Figura 2 muestra la interface del modelo Chancado y Molienda, se aprecia los equipos y la plataforma para ingresar las variables de entrada del modelo. 


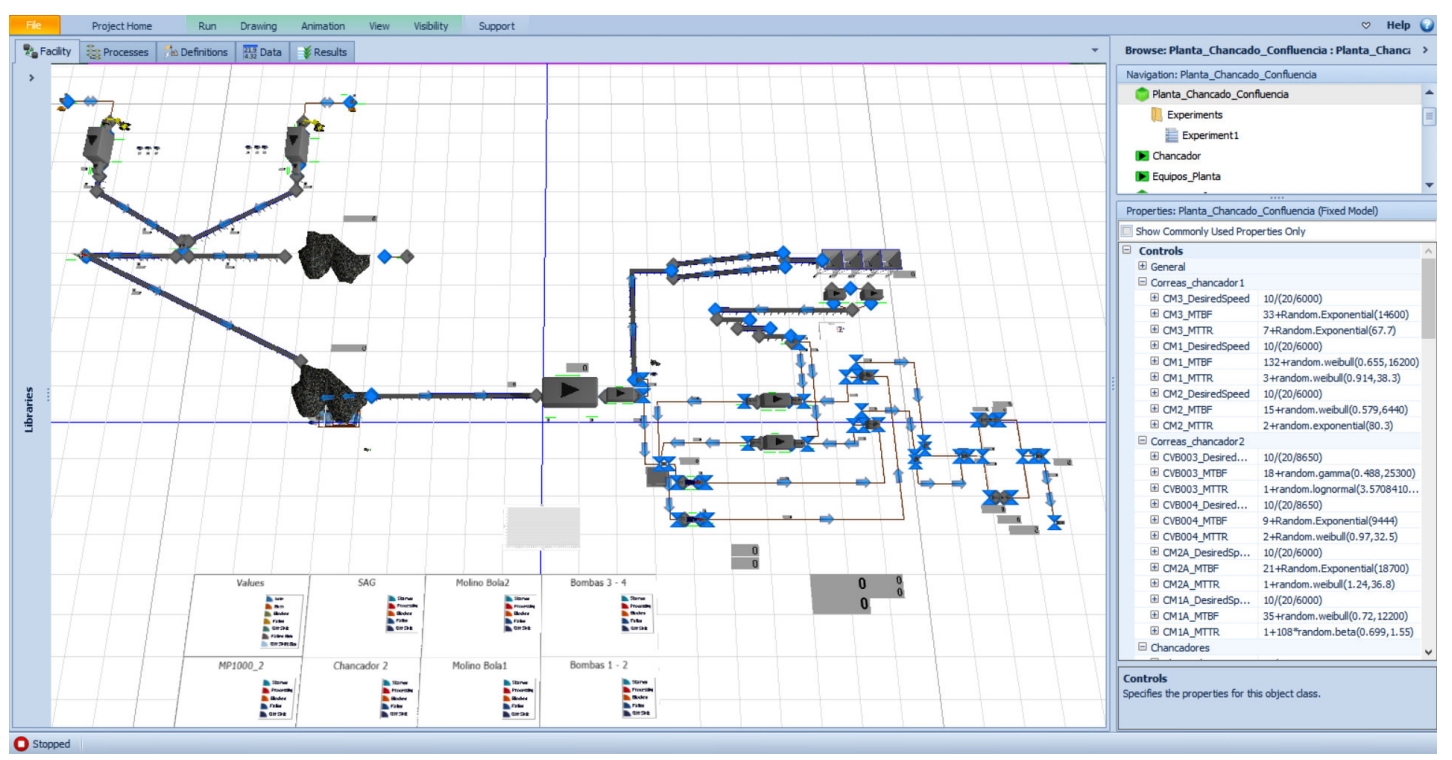

Figura 2. Animación del modelo de simulación, SIMIOß.

\section{Inputs del modelo}

Las detenciones programadas de los equipos principales se ingresan mediante calendario y considera los turnos de trabajos y períodos de mantención.

Los parámetros de entrada considerados son los siguientes:

Capacidad de equipos, estanques y correas; velocidades de correas trasportadoras; caudales de alimentación de mineral a equipos; detenciones programadas por calendario; detenciones no programadas (variables aleatorias TBF y TTR).

\section{Outputs del modelo}

Los datos de variables de resultados de los principales equipos se obtienen por medio de planillas Excel@. Las variables de salida consideradas son los siguientes: producción de mineral en toneladas por día (KTPD); tiempos de: procesamiento, fallas, mantenciones programadas, sin alimentación y bloqueado; utilización; nivel de estanques y tolvas.

\section{RESULTADOS}

A continuación se presenta la descripción de los resultados de la verificación y validación del modelo y los resultados experimentales.

\section{Verificación y Validación del modelo}

Para el modelo de simulación computacional logrado se verifica que el comportamiento efectivamente imite el comportamiento real, con la participación de personal de operaciones de la empresa, se realizan pruebas de verificación cualitativas del comportamiento de las variables de salida, demostrando satisfactoriamente la eficacia del modelo computacional.

La validación se aborda evaluando la variable de resultado Producción de la Planta, comparando con los datos reales del 2014. La simulación arrojó una producción total media de 34.523.063 toneladas de mineral seco, con un intervalo de confianza (95\%) entre [34.301.580; 34.697.560] toneladas de mineral seco al cabo del 2014, versus las 34.306.904 toneladas reales obtenidas el mismo año. Esto significa que la simulación entrega un error relativo de $0,66 \%$ y un error porcentual absoluto medio del $2,67 \%$ para el período analizado. Respecto a la señal de seguimiento del error de pronóstico, el error se encuentra bajo control dentro de los límites de \pm 3 Desviaciones Absoluta Media (DAM), tal como se muestra en la Figura 3.

\section{EXPERIMENTACIÓN}

\section{Línea base "cuellos de botella"}

Experimento para responder la pregunta ¿cuáles son los equipos "cuellos de botella" de la Planta en la situación actual de operación? 


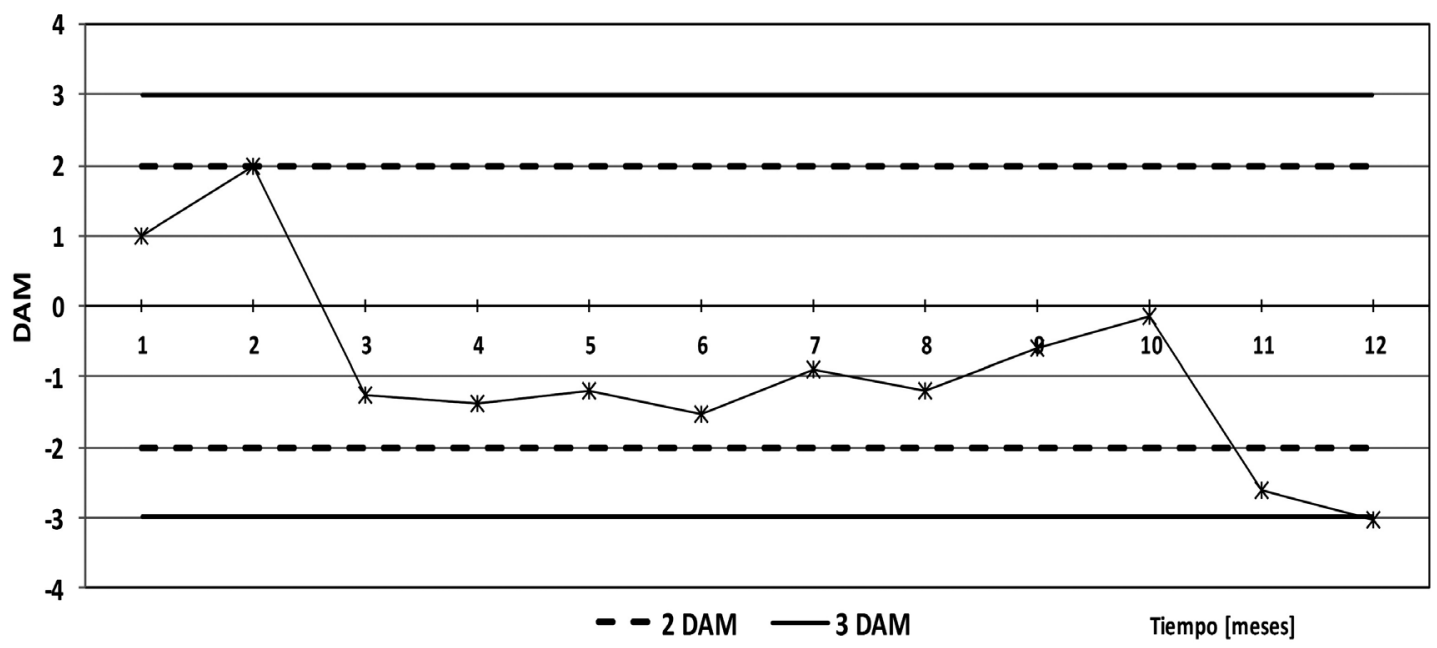

Figura 3. Señal de seguimiento de error de pronóstico de la producción mensual de la Planta, 2014.

En base a 10 corridas de simulación de la Planta, se obtienen los estadísticos de media e intervalo de confianza de la estimación, para la variable Utilización en cada equipo. La Figura 4 muestra el porcentaje de utilización promedio y sus respectivos intervalos de confianza por equipo. El Molino SAG presenta la mayor utilización del sistema con $88,6 \%$. El intervalo de confianza de la utilización del SAG no se superpone con los intervalos de confianza del resto de los equipos, por tanto, existe evidencia estadística de que es la mayor utilización global y, en efecto, representa el "cuello de botella" primario de acuerdo con las condiciones actuales de operación de la Planta. El conjunto Bombas 1-2 (que alimenta el conjunto de ciclones 1), posee la segunda utilización más alta. Por último, los chancadores de Pebbles 1 y 2 tienen la menor utilización con $55 \%$ y $7 \%$, respectivamente.

\section{Experimento 1: Variación de alimentación molienda SAG}

Este experimento, para responder la pregunta: ¿cuáles son los equipos "cuellos de botella" de la Planta para distintos niveles de alimentación de mineral a la molienda SAG?, toma como único factor la variable Alimentación del Molino SAG, y simula el comportamiento de la Planta para seis niveles de toneladas por hora, haciendo 10 corridas en cada nivel.

El porcentaje de utilización promedio de cada equipo en los seis niveles evaluados, se muestra en

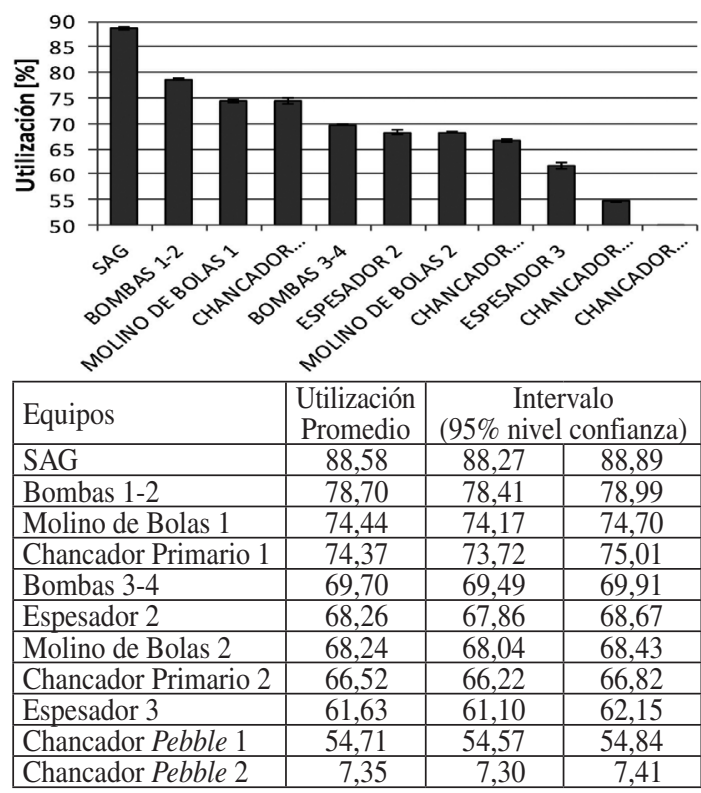

Figura 4. Utilización equipos de la Planta, escenario actual operación.

la Figura 5. Se advierte que el Molino SAG es el "cuello de botella" principal del sistema, debido al mayor porcentaje de utilización, para todos escenarios de alimentación a la molienda SAG.

El Molino SAG aumenta su utilización, a medida que debe procesar mayor cantidad de mineral, además se observa que el incremento en la alimentación a la molienda SAG es directamente proporcional al aumento en la utilización del Chancador primario 2, 


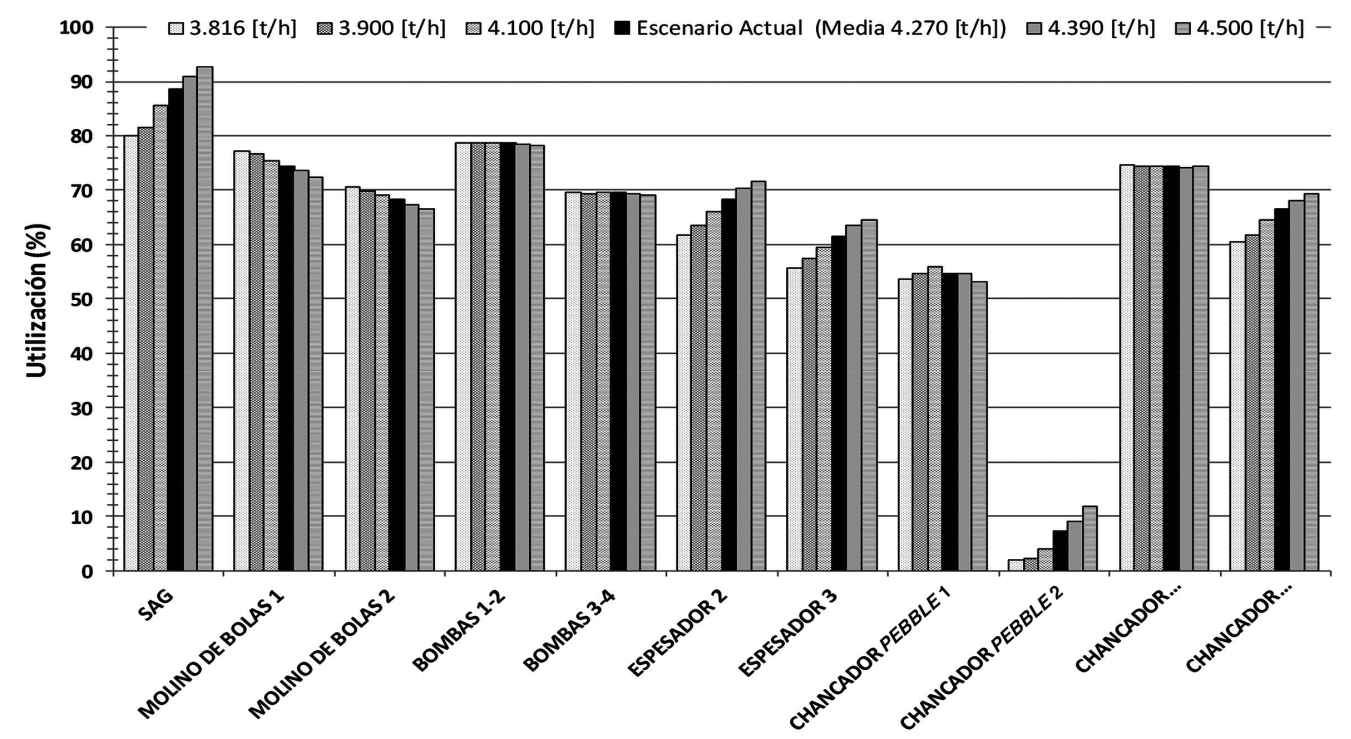

Figura 5. Utilización de equipos en distintos escenarios de alimentación al SAG.

debido al mayor requerimiento de mineral en la molienda SAG. El Chancador primario 1 no se ve afectado, dado que se destina principalmente a abastecer de mineral el Acopio B, fuera de la Planta.

\section{Experimento 2: Variación de Pebbles circulante} Para responder la pregunta: ¿cómo afecta la proporción de Pebbles circulantes en la aparición de "cuellos de botellas"?, el experimento considera como único factor la variable \%Pebbles circulante, y simula el comportamiento de la Planta en seis niveles de porcentaje, haciendo 10 corridas en cada nivel.
Se puede observar en la Figura 6 que el Molino SAG es el "cuello de botella" principal del sistema cuando el porcentaje de Pebbles circulante es menor a 25\%. Para 25\% de Pebbles circulante, el Molino SAG sigue siendo el "cuello de botella" principal con un $88 \%$ de utilización; sin embargo, los chancadores de Pebbles aumentan su utilización al $85 \%$. Para $30 \%$ de Pebbles circulante, la restricción principal del sistema cambia hacia los chancadores de Pebbles, con $90 \%$ de utilización, y el molino SAG pasa a ser el "cuello de botella" secundario con $83 \%$ de utilización.

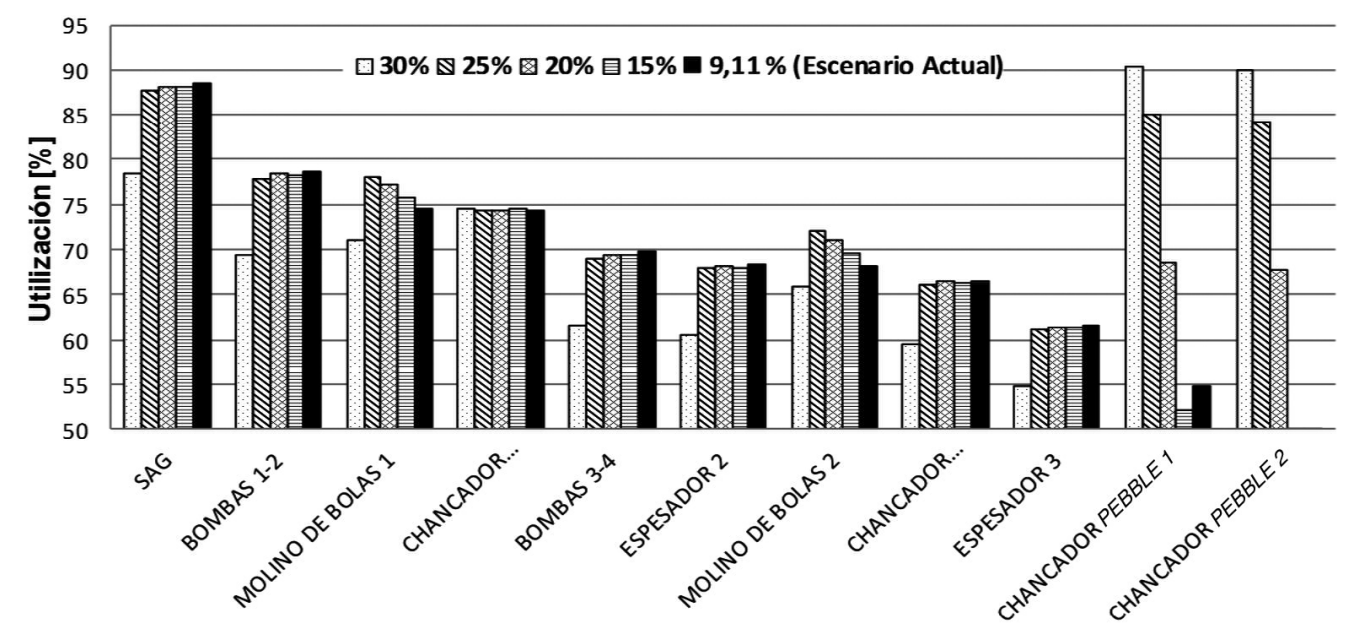

Figura 6. Utilización de equipos en distintos escenarios de porcentajes de Pebbles circulantes. 


\section{Experimento 3: Variación de capacidad chancadores}

El relación a la pregunta: ¿cómo cambia la situación de los equipos "cuellos de botella" producto de variaciones en la capacidad de los chancadores primarios?, el experimento asume como único factor la Capacidad del chancador primario, y simula el comportamiento de la Planta en siete niveles de capacidad en $[\mathrm{t} / \mathrm{h}]$, haciendo 10 corridas por nivel.

Se puede observar en la Figura 7, que el Molino SAG es el "cuello de botella" principal del sistema, incluso para escenarios adversos, donde los chancadores reducen su capacidad hasta $4.000[\mathrm{t} / \mathrm{h}]$.

Para capacidad de procesamiento bajo 4.000 [t/h], la utilización del Molino SAG disminuye considerablemente hasta dejar de ser el "cuello de botella" principal. En un escenario de 3.700 [t/h] de capacidad de chancado, el "cuello de botella" principal se traslada aparentemente al Chancador Primario 1 con $74,9 \%$ de utilización, seguido por el Molino SAG con 74,8\%. Sin embargo, los intervalos de confianza para la estimación de utilización de ambos equipos se sobreponen y, por lo tanto, no existe suficiente evidencia estadística para decidir cuál es el "cuello de botella". En este caso, se puede asegurar que ambos equipos restringen en igual magnitud al sistema. La tercera utilización más elevada corresponde al Chancador Primario 2 con $73 \%$ de utilización y un intervalo de confianza que no se superpone con el de otros equipos.

\section{CONCLUSIONES}

El trabajo permitió lograr un modelo de simulación computacional que replica satisfactoriamente el comportamiento real de la Planta y de los principales equipos del sistema. La dificultad del modelamiento radica en la imitación de la aleatoriedad del sistema, se realizó un complejo levantamiento de los datos que gobierna el sistema, y el consecuente tratamiento estadístico para generar las distribuciones de las variables aleatorias correspondientes.

El método aplicado para la detección de "cuellos de botella", permitió conocer el estado general de utilización promedio de los equipos en el horizonte de tiempo simulado de 365 días y responder a las necesidades de conocimiento acerca del comportamiento de las limitantes de operación del sistema.

El estudio busca generar un aporte a la industria chilena de la Gran Minería del Cobre, dado que existen escasos estudios publicados de DES, aplicado a esta industria, tanto a nivel nacional como internacional.

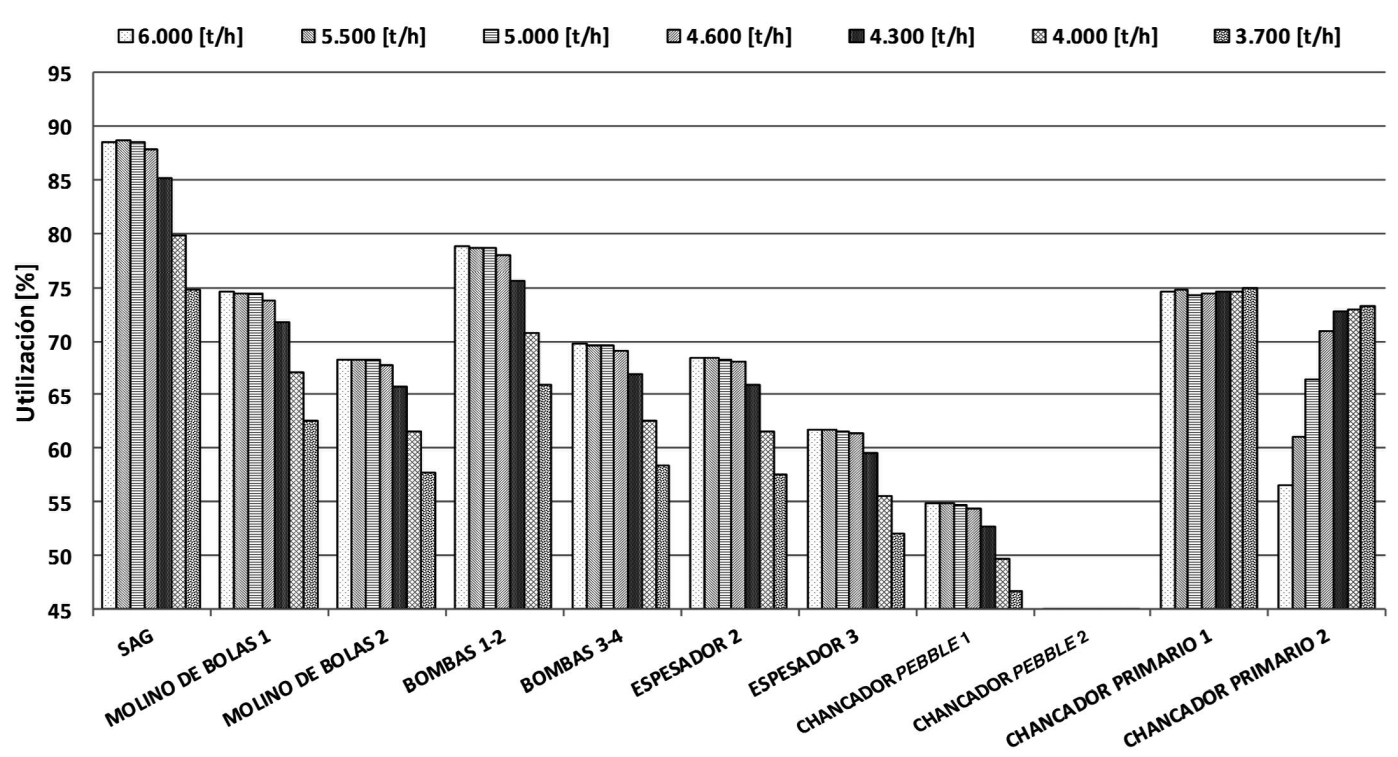

Figura 7. Utilización de equipos en distintos escenarios de capacidad de chancadores primarios. 


\section{AGRADECIMIENTOS}

Este trabajo se enmarca dentro del Proyecto Interno de Investigación N ${ }^{\circ} 251438$ DGIP-UTFSM. Se agradece el aporte derivado del Trabajo de Titulación de Ingeniería Civil MecánicaUTFSM, del señor Johann Alexander Godoy Garvs, septiembre de 2015.

\section{REFERENCIAS}

[1] R.G. Ingalls. "Introduction to simulation". In Simulation Conference. WSC 2008. Winter, pp. 17-26. 2008.

[2] W.D. Kelton, J.S. Smith and D.T. Sturrock. "Simio and Simulation: Modeling, Analysis, Applications". Createspace Independent Pub. ed. 3. 2013. ISBN: 9781493616206.

[3] K.P. White, Jr. and R.G. Ingalls. "Introduction to simulation". In Simulation Conference (WSC). Proceedings of the 2009 Winter, pp. 12-23. Savannah, Georgia, USA. 2009.

[4] S. Bandyopadhyay and R. Bhattacharya. "Introduction to Simulation". Discrete and Continuous Simulation: Theory and Practice, pp. 1-16. CRC Press. 2014.

[5] S.S. Kuo, E.J. Chen, P.L. Selikson and Y.M. Lee. "Modeling continuous flow with discrete-event simulation". In Simulation Conference. 2001. Proceedings of the Winter. Vol. 2, pp. 1099-1103. 2001.

[6] O. Özgün and Y. Barlas. "Discrete vs. continuous simulation: When does it matter". In Proceedings of the 27th international conference of the system dynamics society, pp. 1-22. 2009.

[7] R. Stewart. "A Framework for Simulation Conceptual Modeling". Conceptual Modeling for Discrete-Event Simulation, pp. 73-101. CRC Press. 2010.

[8] V. Bechard and N. Cote. "Simulation of mixed discrete and continuous systems: An iron ore terminal example". In Simulation Conference (WSC). Washington, DC. USA, pp. 1167-1178. Winter, 2013.

[9] M.M. Fioroni, L.A.G. Franzese, C.E. Zanin, J. Furia, L. de Toledo Perfetti, D.Leonardo and N.L. da Silva. "Simulation of continuous behavior using discrete tools: Ore conveyor transport". In Simulation Conference, pp. 1655-1662. Winter, 2007.
[10] A.M. Law. "Designing a simulation study: how to conduct a successful simulation study". In Proceedings of the 35 th conference on Winter simulation: driving innovation, pp. 66-70. New Orleans, Louisiana, USA. 2003.

[11] A.M. Law and M.G. McComas. "Building valid models: how to build valid and credible simulation models". In Proceedings of the 33nd conference on Winter simulation, pp. 22-29. Arlington, Virginia, USA. 2001.

[12] C. Roser, M. Nakano and M. Tanaka. "Comparison of bottleneck detection methods for AGV systems". In Simulation Conference, 2003. Proceedings of the 2003 Winter. Vol. 2, pp. 1192-1198. 2003.

[13] S. Sengupta, K. Das and R.P. VanTil. "A new method for bottleneck detection". In Simulation Conference, 2008. WSC 2008, pp. 1741-1745. Winter, 2008.

[14] M. Dewa and L. Chidzuu. "Managing Bottlenecks in Manual Automobile Assembly Systems using Discrete Event Simulation". South African Journal of Industrial Engineering. Vol. $24 \mathrm{~N}^{\circ} 2$, pp. 155166. 2013. ISSN: $1012277 \mathrm{X}$.

[15] M.K. Williams. "Using Simulation to Understand Bottlenecks, Delay Accumulation, and Rail Network Flow". In Proceedings of the Annual AREMA Conference. 2011.

[16] B. Sharda and S.J. Bury. "Bottleneck analysis of a chemical plant using discrete event simulation". In Simulation Conference (WSC). Proceedings of the 2010 Winter, pp. 1547-1555. 2010.

[17] C. Roser, M. Nakano and M. Tanaka. "Shifting bottleneck detection". In Simulation Conference, 2002. Proceedings of the Winter. Vol. 2, pp. 1079-1086. 2002.

[18] E. Lima, L. Chwif and M. R. Pereira. "Metodology for selecting the best suitable bottleneck detection method". In Simulation Conference, 2008. WSC 2008, pp. 17461751. Winter, 2008.

[19] R.M. Thiesing and C.D. Pegden. "Introduction to Simio". In Proceedings of the 2013 Winter Simulation Conference: Simulation: Making Decisions in a Complex World, pp. 40524061. 2013.

[20] SIMIO. Simio Reference Guide. 2006. Fecha de Consulta: 10/08/2015. URL: http://www. simio.com/academics/student-resources.php 
[21] R.J. Coelho, P.F. Lana, A.C. Silva, T.F. Santos, M.M. Fioroni, L.A.G. Franzese, D. de Oliveira Mota and L.B. da Silva. "Operational simulation model of the raw material handling in an integrated steel making plant". In Simulation Conference (WSC). Proceedings of the 2009 Winter, pp. 3055-3065. 2009.

[22] A.M. Law. "Statistical analysis of simulation output data: the practical state of the art". In Proceedings of the Winter Simulation Conference, pp. 65-74. Baltimore, Maryland. 2010.
[23] M.K. Nakayama. "Output analysis: analysis of simulation output". In Proceedings of the 35th conference on Winter simulation: driving innovation, pp. 49-58. New Orleans, Louisiana, USA. 2003.

[24] J. Heizer and B. Render. "Dirección de la producción y de operaciones: decisiones estratégicas". Pearson Educación. Ed. 8, pp. 616. Madrid, España. 2007. ISBN: 9788483223604. 